Discrete Comput Geom 33:223-229 (2005)

DOI: $10.1007 / \mathrm{s} 00454-004-1142-2$

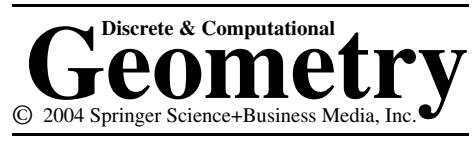

\title{
A Note on Caterpillar-Embeddings with No Two Parallel Edges
}

\author{
Daniel J. Kleitman and Rom Pinchasi \\ Department of Mathematics, \\ Massachusetts Institute of Technology, \\ Cambridge, MA 02139-4307, USA \\ \{djk,room\}@math.mit.edu
}

\begin{abstract}
Let $G$ be a set of $n$ points in general position (i.e., no three points are on a line) in the plane, and let $C$ be a caterpillar on $n$ vertices. We show that one can always find a rectilinear embedding of $C$ in the plane such that the vertices of $C$ are the points of $G$ and no two edges of $C$ go to parallel segments. This proves a conjecture of Robert E. Jamison.
\end{abstract}

\section{Introduction}

Let $G$ be a set of $n$ points in the plane. A direction path for $G$ is a path whose vertices are the points of $G$ and whose edges consist of straight line segments no two of which are parallel (see, for example, Fig. 1). Clearly, a necessary condition for the existence of a direction path for a set $G$ is that the points of $G$ determine at least $n-1$ different slopes.

A well-known theorem of Ungar [U] asserts that any set of $n$ points, which is not contained in a line, determines at least $n-1$ different directions. In [J2] Jamison used this result to show that any non-collinear set of $n$ points in the plane admits a direction tree, namely, a tree whose vertices are the $n$ points, and every pair of edges have different directions. In the same paper (and also in [J1]) Jamison conjectured that if $G$ is a set of $n$ points in general position, then not only that it admits a direction tree, but in fact a direction path. On the other hand, Jamison [J1] constructed arbitrary large non-collinear sets $G$ such that every line determined by $G$ contains at most $|G| / 3+3$ points, but still $G$ does not admit a direction path.

In this paper we prove a generalization of the conjecture of Jamison. We show that his conjecture is in fact true for any caterpillar, as follows:

Theorem 1.1. Let $C$ be any given caterpillar on $n$ vertices, and let $G$ be any set of $n$ points in general position in the plane, then there is a rectilinear embedding of $C$ in the 


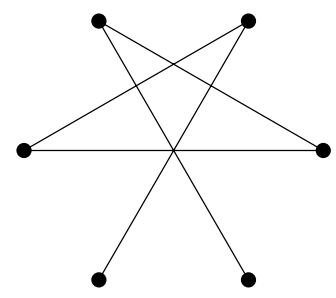

Fig. 1. A directed path for the set of vertices of a regular hexagon.

plane so that the vertices of $C$ are the points of $G$ and every pair of edges of $C$ have different directions.

We note that a special case of Theorem 1.1, where $G$ is the set of vertices of a regular $n$-gon, was shown by Jamison. There it follows from the equivalence of a tree being a direction tree for the set of vertices of a regular $n$-gon, and a labeling of the vertices of a tree by the numbers $0,1, \ldots, n-1$ so that the sums of the labels (modulo $n$ ) of every two adjacent vertices of the tree are pairwise different.

Pach $[\mathrm{P}]$ observed that the proof presented in this paper is similar to a proof presented in $\left[\mathrm{AGH}^{+}\right]$(in its dual version) for bipartite embedding of paths in the plane with no two edges crossing. Indeed, Pach managed to show that Jamison's conjecture for a path can be shown to be implied by the theorem in $\left[\mathrm{AGH}^{+}\right]$. Nevertheless, the proof presented here applies to general caterpillars and to arrangements of $x$-monotone pseudolines rather than just points in the plane. At the same time, however, the proof presented here can be used to prove an extension of the theorem in $\left[\mathrm{AGH}^{+}\right]$to general caterpillars as well.

\section{Caterpillars}

In this section we prove Theorem 1.1. We use duality of points and lines by which Theorem 1.1 will follow from the following theorem on arrangement of lines (in fact, everything remains true if we consider arrangements of $x$-monotone pseudolines).

We say that an arrangement of lines $\mathcal{A}$ in the plane is in general position if no three lines of $\mathcal{A}$ pass through the same point, and no line in $\mathcal{A}$ is vertical. We also require that every two lines in $\mathcal{A}$ cross (that is, no two lines in $\mathcal{A}$ are parallel).

Theorem 2.1. Let $\mathcal{A}$ be an arrangement of $n$ lines in general position in the plane. Let $C$ be any caterpillar on $n$ vertices. Then one can find a correspondence between the lines of $\mathcal{A}$ and the vertices of $C$ so that those intersection points of (pairs of) lines which correspond to adjacent vertices in $C$ have pairwise different $x$-coordinates.

It is easy to see how Theorem 1.1 follows from Theorem 2.1. Indeed, let $G$ be a set of $n$ points in general position in the plane. Fix a coordinate system in the plane. By a suitable rotation of the set $G$ we can assume that no two points of $G$ have the same 
$x$-coordinates. Apply the points-lines duality which takes a point $(A, B)$ to the lines $y+A x+B=0$, and takes a (non-vertical) line $y+A x+B=0$ to the point $(A, B)$. Observe that by this duality parallel lines go to points with the same $x$-coordinate and vice versa.

The set $G$ is then transfered to an arrangement of lines in general position. If we now apply Theorem 2.1 to this arrangement, we get exactly what we want. Indeed, embed the caterpillar $C$ in the plane by sending the vertices of $C$ to the dual points of their corresponding lines from Theorem 1.1. If $l_{1}, l_{2}$ are two lines in the plane which contain two different embedded edges of $C$, then the dual of $l_{1}$ and $l_{2}$ will be two points with different $x$-coordinates in the dual plane (according to the result in Theorem 1.1). This means that $l_{1}$ and $l_{2}$ are not parallel.

Instead of proving Theorem 2.1 directly, we will prove a slightly stronger version that will be required for the induction argument. We need a bit of terminology and notation.

Let $C$ be a caterpillar. We say that $C$ has type $(a, b)$ where $a$ and $b$ are positive integers, if, as a bipartite graph, $C$ has its two color classes with cardinalities $a$ and $b$, respectively. We then write $V(C)=\left(V_{1}, V_{2}\right)$ for the set of vertices of $C . V_{1}$ and $V_{2}$ are the two color classes of $C$ as a bipartite graph, and of course $\left|V_{1}\right|=a$ and $\left|V_{2}\right|=b$. The vertices of $C$ are divided into two kinds, the leaves and those vertices that are not leaves which we call links. In a caterpillar the vertices which are links form a path which we call the spine. A vertex of $C$ is called a head if it is an extreme link of the spine.

We say that an arrangement $\mathcal{A}$ of lines is of type $(a, b)$ if it consists of precisely $a$ lines with positive slopes and $b$ lines with negative slopes. We denote $\mathcal{A}=\left(L^{+}, L^{-}\right)$ where $L^{+}$is the set of lines with positive slopes and $L^{-}$is the set of lines with negative slopes.

We are now ready to state the stronger version of Theorem 2.1. (The statement of the theorem is a bit long but very easy to understand.) In this theorem we let $C$ be a caterpillar of type $(a, b)$, we let $V(C)=\left(V_{1}, V_{2}\right)$, and we assume that $V_{1}$ contains a vertex $v$ which is a head of $C$. Those assumptions are not restrictive because if $C$ does not have any head, then it consists of only one edge, in which case everything is trivial. Once we know that $C$ has a head we may assume it is in $V_{1}$ for otherwise we reflect the whole scene about the $x$-axis (as we do when applying the induction hypothesis, see details below).

Theorem 2.2. Let $C$ be a caterpillar of type $(a, b)$, and let $V(C)=\left(V_{1}, V_{2}\right)$. We assume that $V_{1}$ contains a vertex $v$ which is a head of $C$. Let $\mathcal{A}=\left(L^{+}, L^{-}\right)$be an arrangement of lines of type $(a, b)$ in general position in the plane. Assume that there is a point $p$ on precisely one of the lines in $L^{+}$such that every line from $L^{-}$passes above $p$ and every line in $L^{+}$passes through or below $p$. Then one can find a one to one correspondence between the lines in $L^{+}$and the vertices in $V_{1}$ and a one to one correspondence between the lines in $L^{-}$and the vertices in $V_{2}$, such that the following is true:

1. The $x$-coordinates of the intersection points of pairs of lines that correspond to adjacent vertices in $C$ are pairwise different and strictly greater than the $x$ coordinate of $p$. 
2. If $l$ is any line with negative slope that passes through $p$, then the $x$-coordinate of the intersection point of $l$ with the line $l_{v}$, that corresponds to $v$, is smaller than the $x$-coordinate of the intersection point of any two lines $l_{1}, l_{2} \in \mathcal{A} \backslash\left\{l_{v}\right\}$ which correspond to two adjacent vertices in $C$.

Proof of Theorem 2.2. We start with an easy observation.

Claim 2.3. Let $p^{\prime}$ be any intersection point of a line from $L^{+}$with a line from $L^{-}$. Then the $x$-coordinate of $p^{\prime}$ is strictly larger than that of $p$.

Proof. Indeed, if $l^{+} \in L^{+}$and $l^{-} \in L^{-}$, then we know that $l^{-}$passes above $p$ and $l^{+}$ passes through or below $p$. Since the slope of $l^{+}$is larger than that of $l^{-}, l^{+}$is always below $l^{-}$, when we are to the left of $p$. In particular, $p^{\prime}$, the intersection point of $l^{+}$and $l^{-}$, must be to the right of $p$.

If $a=1$, then $C$ is a star. We denote by $l_{v}$ the only line in $L^{+}$, and assign it to the vertex $v$. We arbitrarily assign the lines in $L^{-}$to the $b$ vertices in $V_{2}$. Clearly, the intersection points of $l_{v}$ with the lines in $L^{-}$have pairwise different $x$-coordinates (since $\mathcal{A}$ is in general position and thus no three lines of it pass through the same point). Moreover, by Claim 2.3, all these intersection points have their $x$-coordinates greater than that of $p$. Assertion 2 in the theorem is, in this case, void, since one of every two adjacent vertices in $C$ must be $v$. Therefore we are done.

Thus we may assume that $a>1$ (and hence there is a link of $C$ in $V_{2}$ ). Let $m \geq 1$ denote the degree of $v$ in $C$. Since $v$ is a head of $C$ and $a>1$, vertex $v$ has $m-1$ neighbors which are leaves and another neighbor which is a link.

For every line $l \in L^{+}$, let $z_{1}^{l}, \ldots, z_{m}^{l}$ denote the $x$-coordinates of the $m$ leftmost intersection points of $l$ with lines from $L^{-}$. Let $l_{v}$ be such that $z_{m}^{l_{v}}=\min _{l \in L^{+}}\left\{z_{m}^{l}\right\}$. Let $p_{v}$ denote that (intersection) point on $l_{v}$ whose $x$-coordinate is $z_{m}^{l_{v}}$. Let $l_{1}^{-}, \ldots, l_{m-1}^{-}$ denote the lines from $L^{-}$that intersect $l_{v}$ at the points with $x$-coordinates $z_{1}^{l_{v}}, \ldots, z_{m-1}^{l_{v}}$, respectively.

The idea of the proof is to assign the line $l_{v}$ to $v$ and the lines $l_{1}^{-}, \ldots, l_{m-1}^{-}$arbitrarily to the $m-1$ neighbors of $v$ that are leaves, and then conclude by the induction hypothesis on the remaining caterpillar after removing $v$ and its $m-1$ neighbors that are leaves. However, we first have to make sure that this assignment satisfies the requirements in the theorem and that the conditions of the theorem are satisfied when applying induction. This is the aim of the following claims.

\section{Claim 2.4.}

(1) Every line of $L^{-} \backslash\left\{l_{1}^{-}, \ldots, l_{m-1}^{-}\right\}$passes through or above $p_{v}$.

(2) Every line of $L^{+} \backslash\left\{l_{v}\right\}$ passes below $p_{v}$.

Proof. (1) is obvious, since if $l^{-} \in L^{-} \backslash\left\{l_{1}^{-}, \ldots, l_{m-1}^{-}\right\}$passes below $p_{v}$, then there are $m$ lines from $L^{-}$, namely, $l_{1}^{-}, \ldots, l_{m-1}^{-}$and $l^{-}$which intersect $l_{v}$ at points with $x$-coordinates smaller than that of $p_{v}$, contradicting the definition of $p_{v}$. 


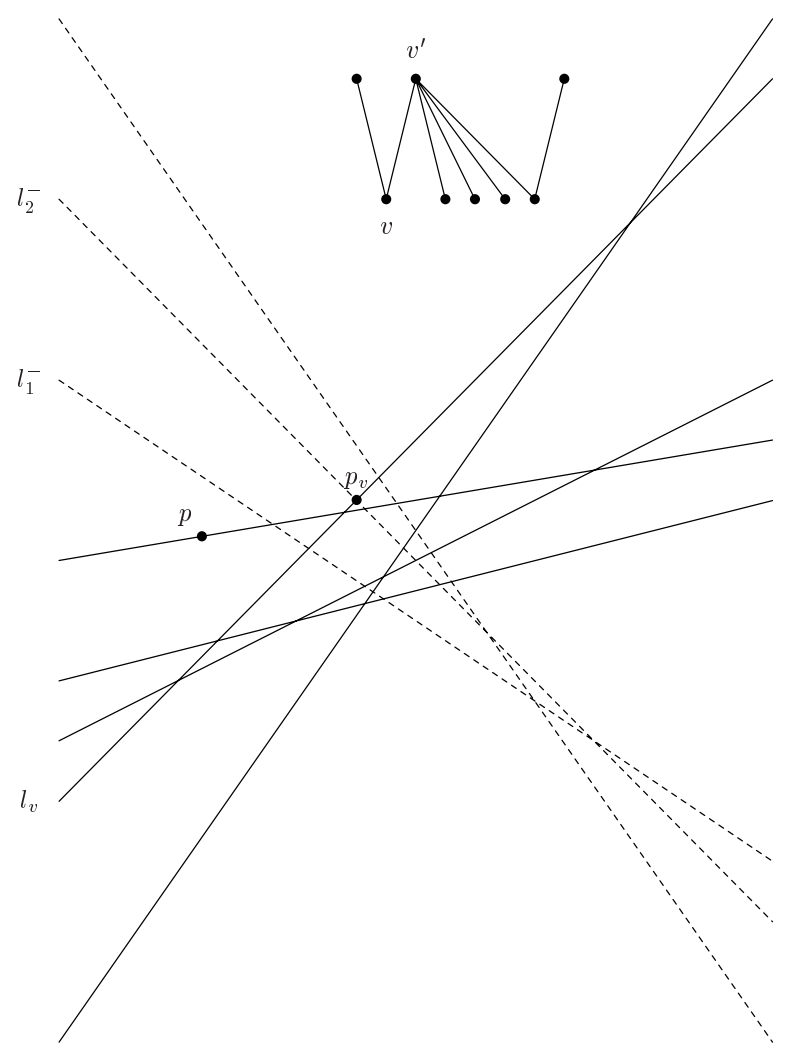

Fig. 2. Determining $p_{v}$ : here $m=2$. A small copy of the caterpillar $C$ is shown on the top. $p_{v}$ and $l_{v}$ as well as $l_{1}^{-}$and $l_{2}^{-}$are indicated in the figure. The dotted lines correspond to lines with negative slopes.

To prove (2), let $l^{+} \in L^{+} \backslash\left\{l_{v}\right\} . l^{+}$cannot pass through $p_{v}$ since $l_{v}$ and a $l_{m}^{-}$pass through it. If $l^{+}$passes above $p_{v}$, then the lines $l_{1}^{-}, \ldots, l_{m}^{-}$intersect $l^{+}$at points with $x$-coordinates smaller than that of $p_{v}$. This would mean $z_{m}^{l^{+}}<z_{m}^{l_{v}}$, contradicting the definition of $l_{v}$.

Claim 2.5. Let $l$ be any line with negative slope through $p$. Let $q$ be the intersection point of $l$ with $l_{v}$. Then the $x$-coordinate of $q$ is greater than or equal to the $x$-coordinate of $p$ and is strictly smaller than the $x$-coordinate of $p_{v}$.

Proof. If $q=p$, then there is nothing to prove since we know, from Claim 2.3, that $p_{v}$ has its $x$-coordinate strictly larger than that of $p$.

Assume then that $q \neq p$. In this case $l_{v}$ passes below $p$ (rather than through $p$, because otherwise $p=q$, the intersection point of $l$ with $l_{v}$ ). Just like in the proof of Claim 2.3, since $l$ passes through $p$ and $l_{v}$ passes below $p$ and the slope of $l$ is smaller than that of $l_{v}$, then $q$ must be to the right of $p$, that is, its $x$-coordinate is greater than that of $p$. 
We now show that the $x$-coordinate of $q$ is strictly smaller than that of $p_{v}$. Let $l_{p}^{+}$ denote the line from $L^{+}$which passes through $p$. We know that $l_{p}^{+} \neq l_{v}$. Assume to the contrary that $q$ (which is the intersection point of $l$ with $l_{v}$ ) is either $p_{v}$ or its $x$-coordinate is larger than the $x$-coordinate of $p_{v}$. Then, since $l$ has negative slope and $l_{v}$ has positive slope, $l$ passes through or above $p_{v}$ (recall that $p_{v}$ lies on $l_{v}$ ). However, $l_{p}^{+}$is above $l$ when we are to the right of $p$ so in particular $l_{p}^{+}$passes above $p_{v}$. This is a contradiction to part (2) of Claim 2.4.

We now conclude the proof of the theorem by using induction (on the size of the caterpillar $C$ ): we assign $l_{v}$ to the vertex $v$ and arbitrarily assign $l_{1}^{-}, \ldots, l_{m-1}^{-}$to the $m-1$ neighbors of $v$ which are leaves.

Let $C^{\prime}$ be the caterpillar of type $(a-1, b-m+1)$ obtained from $C$ by removing the vertex $v$ and its $m-1$ neighbors which are leaves. Denote $V\left(C^{\prime}\right)=\left(V_{1}^{\prime}, V_{2}^{\prime}\right)$. Observe that $V_{2}^{\prime}$ must contain a head of $C^{\prime}$ (namely, the neighbor of $v$ which is a link in $C$ ). Define $L^{\prime+}=L^{+} \backslash\left\{l_{v}\right\}$ and $L^{\prime-}=L^{-} \backslash\left\{l_{1}^{-}, \ldots, l_{m-1}^{-}\right\}$. Clearly, $\left|L^{\prime+}\right|=a-1$ and $\left|L^{\prime-}\right|=b-m+1$. Observe that there is a line in $L^{\prime-}$, namely, $l_{m}^{-}$which passes through $p_{v}$. By Claim 2.4, every line from $L^{\prime+}$ passes below $p_{v}$ and every line from $L^{\prime-}$ passes through or above $p_{v}$.

Now apply the induction hypothesis on the smaller caterpillar $C^{\prime}$ by interchanging the roles of $C_{1}^{\prime}$ and $C_{2}^{\prime}$, so that now there is a head of $C^{\prime}$ in the correct color class. As the arrangement of lines we take $L^{\prime+} \cup L^{\prime-}$ reflected about the $x$-axis. Therefore, the reflection of $L^{\prime+}$ will serve as the set of lines with negative slopes and the reflection of $L^{\prime-}$ will serve as the set of lines with positive slopes. We take the point $p_{v}$ to play the role of $p$ in the statement of the theorem. Let $v^{\prime}$ be the (only) neighbor of $v$ which is a link in $C . v^{\prime} \in V_{2}^{\prime}$ is a head of $C^{\prime}$. We take $v^{\prime}$ to play the role of $v$ in the statement of the theorem.

It is easy to see that all the conditions of the theorem are satisfied by the new caterpillar $C^{\prime}$ and the new reflected arrangement of lines. We can thus find a correspondence between the lines of $L^{\prime+}$ and the vertices of $V_{1}^{\prime}$ and between the lines of $L^{\prime-}$ and the vertices of $V_{2}^{\prime}$. By this correspondence all intersection points between lines which correspond to adjacent vertices in $C^{\prime}$ have pairwise different $x$-coordinates, and they all lie to the right of $p_{v}$ so that they have different $x$-coordinates than those intersection points between $l_{v}$ and $l_{1}^{-}, \ldots, l_{m-1}^{-}$.

We have to consider only one more intersection point between the line $l_{v}$ and the line $l_{v}^{\prime}$ which corresponds to $v^{\prime}$ (recall that $v$ and $v^{\prime}$ are neighbors in $C$ ). Denote that intersection point by $r$. We want to show that the $x$-coordinate of $r$ is different than the $x$-coordinate of the intersection point of any two lines which correspond to adjacent vertices in $C$. Clearly, the $x$-coordinate of $r$ is different than those of the intersection points of $l_{v}$ with any of the lines $l_{1}^{-}, \ldots, l_{m-1}^{-}$. If we take the line $l$ in the statement of the theorem to be the reflection of $l_{v}$ about the $x$-axis (recall that $l_{v}$ passes through $p_{v}$ ), then it follows from the induction hypothesis that $r$ is to the left of any intersection point of two lines $l_{1}, l_{2} \in \mathcal{A} \backslash\left\{l_{v}, l_{v^{\prime}}\right\}$ which correspond to adjacent vertices in $C$. This implies that the $x$-coordinate of $r$ is different from every $x$-coordinate of two lines which correspond to adjacent vertices in $C$ as required.

Finally, we have to show the validity of assertion 2 in the theorem. Let $l$ be any line with negative slope through $p$. Observe that every two lines $l_{1}, l_{2} \in \mathcal{A} \backslash\left\{l_{v}\right\}$, which 
correspond to two adjacent vertices, must be in $L^{\prime+} \cup L^{\prime-}$. By the induction hypothesis, the intersection point of any two lines $l^{+} \in L^{\prime+}$ and $l^{-} \in L^{\prime-}$ which correspond to adjacent vertices in $C^{\prime}$ is to the right of $p_{v}$. However, the intersection point of $l$ and $l_{v}$ is, by Claim 2.5, to the left of $p_{v}$.

Open Problem. It is a striking open problem whether Theorem 1.1 is true if $C$ is any tree on $n$ vertices. This problem is already very interesting in the case where the set of points $G$ is the set of vertices of a regular $n$-gon. Then this problem is equivalent to the so-called Harmonic Tree Conjecture.

\section{References}

$\left[\mathrm{AGH}^{+}\right]$M. Abellanas, J. Garcla, G. Hernandez, M. Noy, and P. Ramos, Bipartite embeddings of trees in the plane. Discrete Appl. Math. 93(2-3) (1999), 141-148.

[J1] R.E. Jamison, Direction paths, Congr. Numer. 54 (1986), 145-156.

[J2] R.E. Jamison, Direction trees, Discrete Comput. Geom. 2 (1987), 249-254.

[P] J. Pach, Personal communication.

[U] P. Ungar, $2 N$ noncollinear points determine at least $2 N$ directions, J. Combin. Theory Ser. A 33 (1982), 343-347.

Received April 17, 2003, and in revised form April 20, 2004. Online publication November 19, 2004. 